\title{
The thermodynamic basis of mechanisms of bacterial virus infection*
}

\author{
G. M. Mrevlishvili ${ }^{1} \dagger$, T. Mdzinarashvili ${ }^{1}$, M. Al-Zaza ${ }^{1}$, L. Tsinadze ${ }^{1}$, \\ D. Tushishvili ${ }^{2}$ and G. Razmadze ${ }^{1}$ \\ ${ }^{1}$ Department of Physics, Tbilisi State University, 380028, Tbilisi, Georgia; ${ }^{2}$ Institute of \\ Bacteriophage, Microbiology \& Virology, Georgian Academy of Science, 380001, Tbilisi, \\ Georgia
}

\begin{abstract}
By using of differential scanning calorimetry (DSC) and temperature scanning rotational viscosimetry the specific phase transition we study in the heated suspensions of phages $\left(\mathrm{S}_{\mathrm{d}}, \mathrm{DDVI}\right)$ modeling the process of injection of the genetic material from the phage: the transition of interphage ds-DNA from the state of intramolecular liquid crystal into the coiled state of ds-DNA. The other conformational rearrangements represent the thermal denaturation of the coat proteins and helix-coil transition in DNA.

We detect that:

1 ds-DNA output from the phage head (capsid), induced by temperature, not accompanied by any thermal (endo-or exo-) heat effects (temperature interval $48-60^{\circ} \mathrm{C}$; DSC sensitivity $0.4 \mu \mathrm{W} / \mathrm{cm})$.

2 ds-DNA output from the capsid, increase of the suspension viscosity at least 100-times (!) and recent experiments reveal that it is not one-step process; we have at least two distinct phases of outlet of genetic material, manifesting, that there are strong interactions between the DNA sites and some proteins inside the capsid. It is not excluded that kinetic factors also regulate this ejection process.

3 According to our working hypothesis the main factors of the DNA condensation and packaging in viruse head and after its ejection through the hole with diameter close to dsDNA's, are caused by the surrounded solution 'quality' and so-called 'hydration forces' between ds-DNA parallel packaged segments and, more exactly, by the difference of this parameters inside and outside the capsid of the phage.
\end{abstract}

\section{INTRODUCTION}

'Are phages (and other viruses) living? Now we realize that all viruses are small pieces of genetic material (DNA or RNA), each enclosed within a protective protein-rich coat that facilitates its transportation from one cell to another. As long as the phage chromosome is tightly enclosed within the capsid (protein coat), it cannot provide the information to direct the synthesis of either new chromosomes or new coat protein molecule' [1].

Except biological there are some interesting problems from physical point of view: How is the genetic material-the double stranded DNA (ds-DNA) packed inside the coat protein shell of the small internal volume? What does the interphage DNA tertiary structure represent and in what thermodynamic state of ds-DNA inside the phage capsid is? What is the main physical mechanism

\footnotetext{
*Lecture presented at the 15th International Conference on Chemical Thermodynamics, Porto, Portugal, 26 July-1 August 1998, pp. 1167-1306.

$\dagger$ Corresponding author: George M. Mrevlishvili, Department of Physics, Tbilisi State University, Chavchavadze av.1, 380028, Tbilisi, Georgia. E-mail: biophys@ hepi.edu.ge
} 
of injection of the bacteriophage genetic material (ds-DNA) into the cell (or surrounding aqueous media)?

We can solve this problem by means of thermodynamic and hydrodynamic techniques studying the whole bacterial viruses $\left(S_{d}, D D V I\right)$ and the phages main subcomponents: the protein coat and the ds-DNA separately in the temperature interval $0-110^{\circ} \mathrm{C}$ (see for example [2,3]).

From X-ray scattering studies $[4,5]$, it is known that the interphage DNA has a highly ordered structure and most its strands are parallel to the neighboring ones. The electron microscopy has shown that gently disrupted capsid nucleic acid is mainly organized in a concentric shells (rings) [6,7] and inside the phage head DNA external layer parts were observed in a concentric circle arcs or a parallel longitudinal arrays [8]. Numerous X-ray and optical investigations $[9,10]$ have confirmed that inside the phage capsid ds-DNA in its sugar phosphate backbone does not include disordered parts and has the B-form-family secondary structure. The packed DNA chemical modification indicating the difference between the structure of interphage DNA and free aqueous DNA [11-13] is explained either by the presence of 'kinking' in the packaged DNA or by the interactions between the nucleic acid with the coat and internal capsid proteins. On the other hand, Raman spectroscopy measurements of P22 virion suggest that non-B form DNA can account for no more than $1 \%$ of their packaged DNA [14].

The DNA arrangement in the phage head as 'spool of thread' [4] explaining the experimental data sufficiently well is thought to be a currently favored model. However, there are alternative models supposing a presense of sharp folds (kinks) in the interphage DNA [15]: the 'nematic liquid crystal' [16] and 'spiral fold' model [17] (see also [18]).

In agreement with the theoretical model $[19,20]$ the ds-DNA inside the capsid represents the persistent chain which is located in spherical cavity in globular state and clear expresses liquid crystal order, interacts with coat proteins and can 'reptate' in surrounding aqueous medium through a hole with diameter close to DNA's one. The remaining in the capsid DNA part keeps the globular state whereas leaving the protein shell DNA duplex is in the coil state. Accordingly, the DNA gradual ejection from the phage head can be considered as the peculiar globule to coil transition.

Hence we will study the specific phase transition in the heated phage suspension (modeling the process of injection of genetic material into the cell): the transition of interphage ds-DNA from the state of intramolecular liquid crystal into the coiled state ds-DNA (Fig. 1). The others transitions represent the thermal denaturation of coat proteins and helix-coil transition in the DNA double helix (Fig. 1).

Therefore the aims of this paper are study of: (i) the cooperative conformational reorganisations taking place in the phage suspension under temperature change including thermoinduced ds-DNA ejection from the phage capsid (Fig. 1). (The DSC is the adequate method for this task). (ii) Detailed hydrodynamic analysis of suspension in the temperature intervals of detected transitions and study of the kinetic behaviour of the viscosity in this interval. This kinetic behaviour is studied by using the specially constructed temperature scanning rotational (Zimm-Crothers type) viscometer. (iii) The formulation of the physical model of the process of ds-DNA injection from phage capsid.

\section{EXPERIMENTAL}

\section{Materials}

The bacteriophage $S_{d}$ of high concentration and purity was obtained as described in [2]. It is well known that this coli-phage represents icosahedr (diameter $=30 \mathrm{~nm}$, length $=24 \mathrm{~nm}$ ) with short tail $(3 \mathrm{~nm})$; protein content $55 \%$; DNA $45 \%$. MW of DNA $47-48 \times 10^{6} \mathrm{Da}$. Concentration of DNA in solution and phages in suspensions determined spectrophotometricaly $(1 \mathrm{mg}$ DNA in $1 \mathrm{~mL}=0.018 \mathrm{OD}$ and $0.024 \mathrm{OD}$, respectively, OD is optical density). Protein determined by Lowry method.

The bacteriophage DDV1 was obtained in glass fermenter (capacity 10L) as described earlier [3]; Concentration and purification were carried out on DEAE cellulose and with use differential centrifugation. The highly purified phage suspensions were obtained by additional ultracentrifugation in $\mathrm{CsCl}$ density gradient followed by dialysis. This phage has more long tail, $125 \mathrm{~nm}$. DNA content $50 \%$. MW of DNA is $110 \times 10^{6} \mathrm{Da}$. 


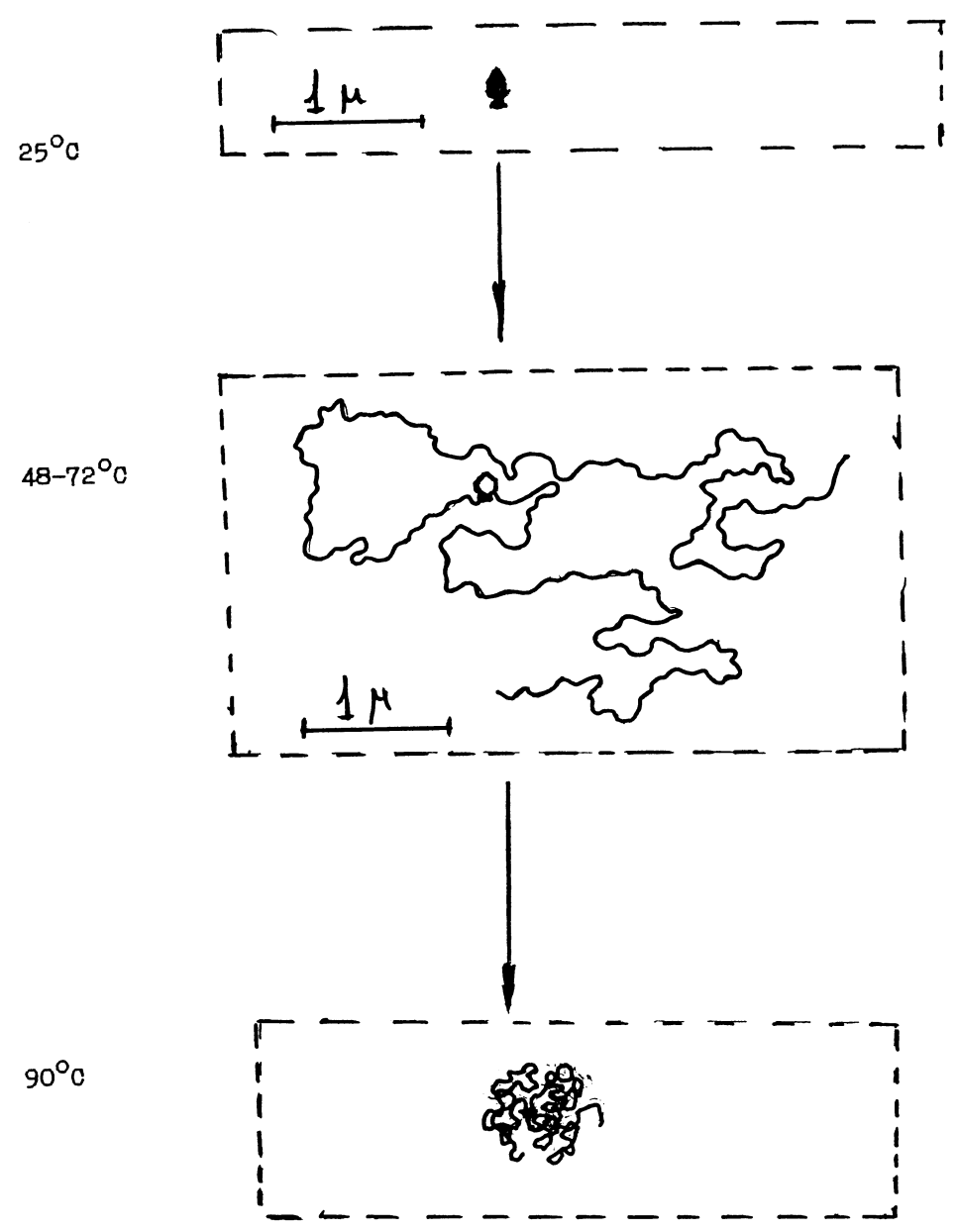

Fig. 1 Diagrammatic representation of conformational rearrangement and phase transitions in bacteriophage $\left(\mathrm{S}_{\mathrm{d}}\right)$ suspensions, based on electron microscopy [23] and hydrodynamic [2] data: $25^{\circ} \mathrm{C}$ native phages; $48-72^{\circ} \mathrm{C}$ release of ds-DNA from phage head: organization of ds-DNA coils with persistent length $\sim 50 \mathrm{~nm}$. $70-82^{\circ} \mathrm{C}$ denaturation of capsid proteins. $90^{\circ} \mathrm{C}$ helix-coil transition in DNA.

\section{METHODS}

The apparent heat capacity $(\mathrm{Cp})$ measurements were carried out using the capillary differential scanning calorimeters DASM-4 and DASM 4A (Poushcino, Russia) [21] with operational volume of the calorimetric cells $0.6 \mathrm{~mL}$ and operational temperature range -10 to $+120^{\circ} \mathrm{C}$. The $\mathrm{Cp}$ measurement error did not exceed $1-2 \%$.

Phage suspension rheological features have been studied by scanning temperature rotation viscometer of Zimm-Crothers type. Continuous heating is achieved by using circulating bath with different heating rates. The value of viscosity is processed in real time by PC through the home built interface. Special programme calculate the value and temperature dependence of the specific viscosity: $\eta_{\mathrm{sp}}=\left(\eta-\eta_{\mathrm{o}}\right) / \mathrm{C} \eta_{\mathrm{o}}$, where $\eta=$ viscosity of solution, $\eta_{0}=$ viscosity of solvent, $C=$ concentration. The working volume of the viscometer was $0.6 \mathrm{~mL}$; the temperature was measured with accuracy $0.2 \mathrm{~K}$; the error of measurement of specific viscosity did not exceed $5 \%$.

\section{RESULTS AND DISCUSSION}

\section{Experimental results}

Figure 2 shows the specific heat capacity (at constant pressure) vs. temperature curve for the phage $S_{d}$ suspension. We see the intensive heat absorption peaks in the three temperature ranges which are 

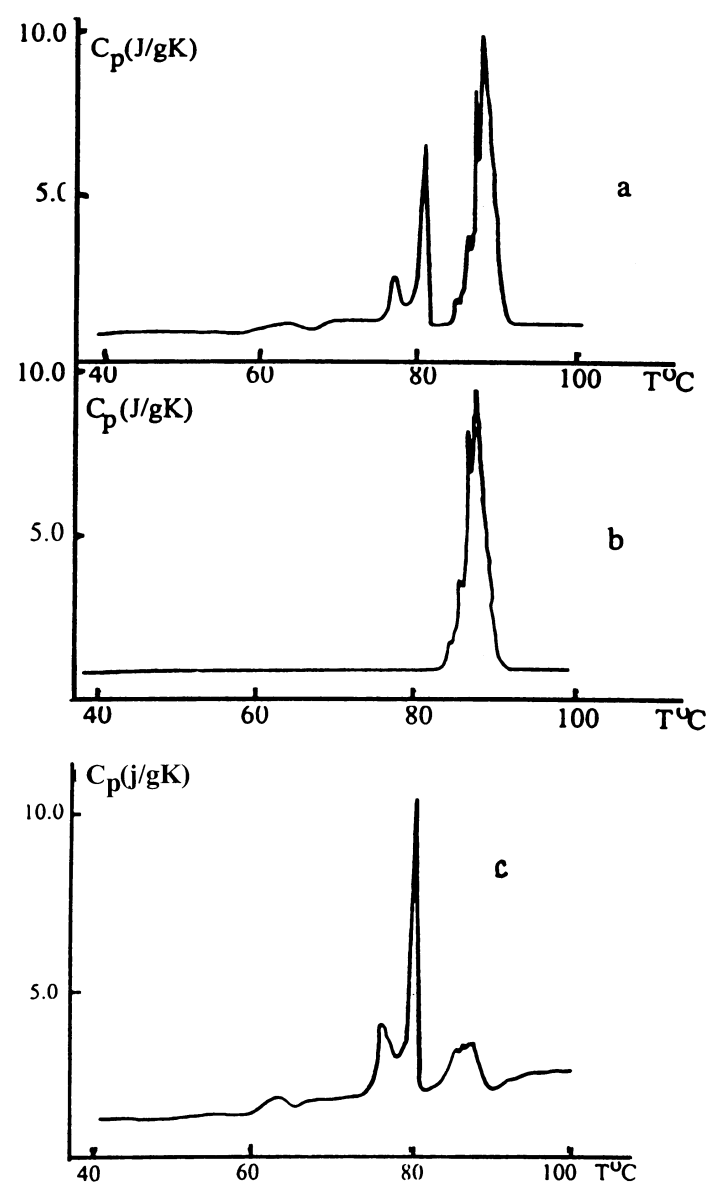

Fig. 2 (a) Temperature dependencies of the heat capacity (Cp) for aqueous suspensions of the bacteriophage $S_{d}$; (a) $(0.15 \mathrm{M} \mathrm{NaCl}+0.015 \mathrm{~m}$ citrate buffer, $\mathrm{pH} 7.0)$. (b) Repetitive heating after cooling the denatured phage.

(c) Temperature dependencies of the heat capacity $(\mathrm{Cp})$ for protein component ('ghost of phages') of phages $S_{d}$ in 1 SSC buffer solutions.

corresponding to: The some structural changes (rearrangement) in the protein capsid framework $\left(60-70{ }^{\circ} \mathrm{C} ; T_{\mathrm{m}}=64^{\circ} \mathrm{C}\right)$. Phage particle protein denaturation $\left(72-85^{\circ} \mathrm{C} ; \Delta \mathrm{H}=24 \pm 2 \mathrm{~J} / \mathrm{g}\right)$. Phage DNA melting $\left(85-95^{\circ} \mathrm{C} ; T_{\mathrm{m}}=88.0^{\circ} \mathrm{C} ; \Delta \mathrm{H}=59 \pm 5 \mathrm{~J} / \mathrm{g}\right)$.

The DNA melting heat absorption peak exhibits the fine structure, typical for this DNA in isolated form [22]. The isolating DNA melting enthalpy and DNA denaturation enthalpy in the composition of phage coincide but the melting temperatures are somewhat different. Essential difference, however, revealed when we study the melting process reversibility: only about $80 \%$ of DNA initial structure are restored after denaturation of isolating DNA in solution, whereas DNA reverses its initial conformation almost completely and restores the fine structure of heat absorption peak in the presence of protein components of viruses (Fig. 2b).

This results allow us to suppose that ligands (such as phage head proteins or polypeptide chain fragments) interacting with ds-DNA in some its sites and preventing from the total unwinding of double helix, however, did not change the DNA secondary structure stabilisation energy.

DSC studies show the absence of any thermal effects in the phage suspension at the temperatures less than $60^{\circ} \mathrm{C}$ (in the interval $20-60^{\circ} \mathrm{C}$ ). However the phage suspension viscosity increases abruptly in the temperature interval $48-60^{\circ} \mathrm{C}$ (Fig. 3). Above given temperature interval corresponds to $S_{d}$ phage genome release according to electron microscopy data [23]. It should be also noted that before DNA reptation process starting the modification of virus particle takes place (e.g. structural changes in basal plate, release from 'cork'). We detect only the heat capacity increase $\left(\approx 64^{\circ} \mathrm{C}\right)$, that probably 


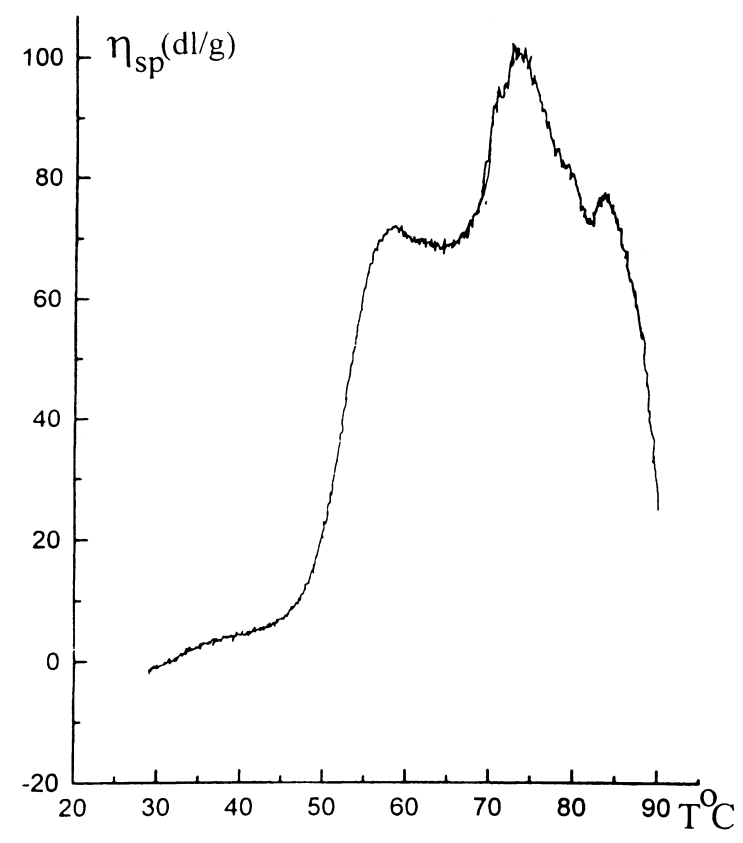

Fig. 3 Temperature dependencies of the characteristic viscosity $\left(\eta_{\mathrm{sp}}\right)$ for aqueous suspensions of the bacteriophage $S_{d}(0.15 \mathrm{M} \mathrm{NaCl}+0.015 \mathrm{~m}$ citrate buffer, $\mathrm{pH} 7.0)$. Scanning rate $\mathrm{V}=0.15 \mathrm{~K} / \mathrm{min}$.

corresponding with the coat protein hydrophobic group-water interactions, because when part of ds-DNA is released from phage head $\left(60^{\circ} \mathrm{C}\right)$ water percolate inside the interior of head and occupies the free volume.

In such a manner, ds-DNA output from capsid, induced by temperature, not accompanied by any thermal (endo- or exo-) heat effects (temperature interval $48-60^{\circ} \mathrm{C}$; DSC sensitivity $0.4 \mu \mathrm{W} / \mathrm{cm}$ ). So the temperature induced 'injection' of genetic material of phages has entropic nature or in this process is totally compensated by the thermal effects of opposite sign. In both cases the Nature selects the way with minimal energetic cost for injection of the genetic material of viruses into the cell.

ds-DNA output from capsid, increase of the viscosity of suspension at least 100-fold (!) and recent rheology experiments reveal that it is not one-step process (see Fig. 3); we have at least two distinct phases of outlet of genetic material $\left(48-60{ }^{\circ} \mathrm{C}\right.$ and $\left.65-72{ }^{\circ} \mathrm{C}\right)$, manifesting that there is a strong interaction between the DNA and some proteins inside the capsid. We underlined this when analysed the process of reversibility after denaturation of the whole phages (see Fig. 2). It is not excluded that kinetic factors also regulate this ejection process (see below). The helix-coil transition in DNA $\left(85-90^{\circ} \mathrm{C}\right)$ naturally accompanied by sharp decreasing of the viscosity (Fig. 3).

Figure 4 represents the temperature dependence of the heat capacity $(\mathrm{Cp})$ and viscosity for the DDV1 phage suspensions at different ionic strengths. As figure shows, intense heat absorption was observed at $86^{\circ} \mathrm{C}$ transcending the temperature region in which the viscosity of the suspension sharply increased $\left(50-60{ }^{\circ} \mathrm{C}\right)$. As we demonstrate increase in the viscosity of bacteriophage suspensions on temperature exposure is linked with reptation ('screeping out') of double helical DNA from the phage head into the surrounding medium. (This is also indicated by electron microscopy study of phages at different temperature [23].) In agreement with data obtained in the suspensions of $S_{d}$ phages, this process of reptation of genetic material from the phage head into solution is not accompanied by thermal effect. As for the destruction of the particles themselves, i.e. the melting of the nucleoprotein complex, in the conditions (1 SSC) indicated this process occurs in the $80-90{ }^{\circ} \mathrm{C}$ interval and (as also to be expected) that is accompanied by a sharp fall in the viscosity associated with unwinding of the double helix and passage of the polynucleotide chains in the statistical coiled state. Increasing in the ionic strength at first led to appearance of an inflection in the heat absorption curve (Fig. 4b) and then to clear separation of the processes of thermal denaturation for double helical DNA and proteins (Fig. 4) (the high temperature peak in both cases refers to the helix-coil transition of DNA and is accompanied by sharp fall in the 

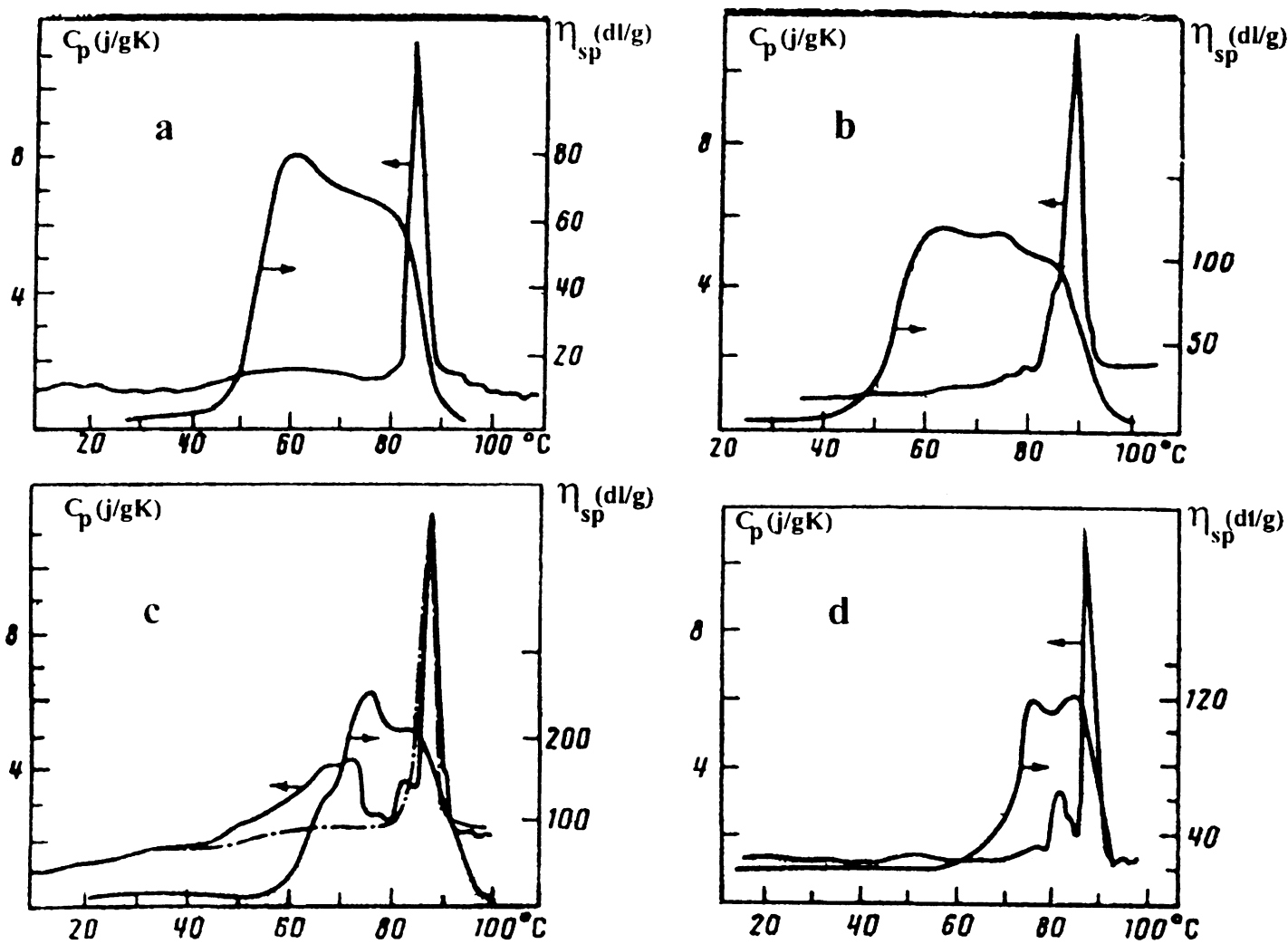

Fig. 4 Temperature dependencies of the heat capacity $(\mathrm{Cp})$ and characteristic viscosity $\left(\eta_{\mathrm{sp}}\right)$ for suspensions of the bacteriophage DDVI for different molarities: (a) 1; (b) 2; (c) 3; (d) 4 SSC. Broken line corresponds to repetitive heating after cooling the denatured phage.

viscosity). It should be noted that the process of thermal denaturation of DNA in the presence of a protein component is fully reversible (Fig. 4c) supporting the existence of a few but quite strong sites of interaction of the proteins and DNA.

Obtained experimental results of temperature-induced phage DNA injection and suggested statisticmechanical model of DNA packaging and reptation from phage $[19,20]$ illustrate that the physical state of surrounding solution and that of filled the capsid one is the dominant factor making the nucleic acid to leave the coat protein shell.

The contraction of the tail of phage plays important role in the injection probably only in the step when phage attaches the outer membrane of host cell (release from 'cork'). The main factors of the DNA condensation and packaging in virus head and after its ejection through the hole with diameter close to DNA's are caused by the solution 'quality' and more exactly by the difference of it inside and outside the shell. The solution 'quality' can particularly reflects such phenomena as: the double helix hydration specificity expressed in hydration layers structural microheterogeneity [24]; the DNA hydration change in transition processes within the double stranded conformation [24]; the various ions influence on the water structure; the DNA and coat protein shell interaction accompanying the reorganisation of hydration layers structure [25], and finally, such phenomenon as the presence of so call 'hydration forces' [26] between interphage DNA parallel packaged segments.

The next problem is: how important the kinetic factors in the regulation of the process of injection of the genetic material of viruses?

We study the hydrodynamic behaviour of the virus suspension inside two temperature intervals, when temperature induced ejection of DNA take place: at temperatures $60^{\circ} \mathrm{C}$ and $70^{\circ} \mathrm{C}$. Figures 5 and 6 present the specific viscosity for phage suspensions vs. time, at 60 and $70^{\circ} \mathrm{C}$, respectively. We see absolutely different kinetical character of reptation of DNA from the phage particle into the solution. 


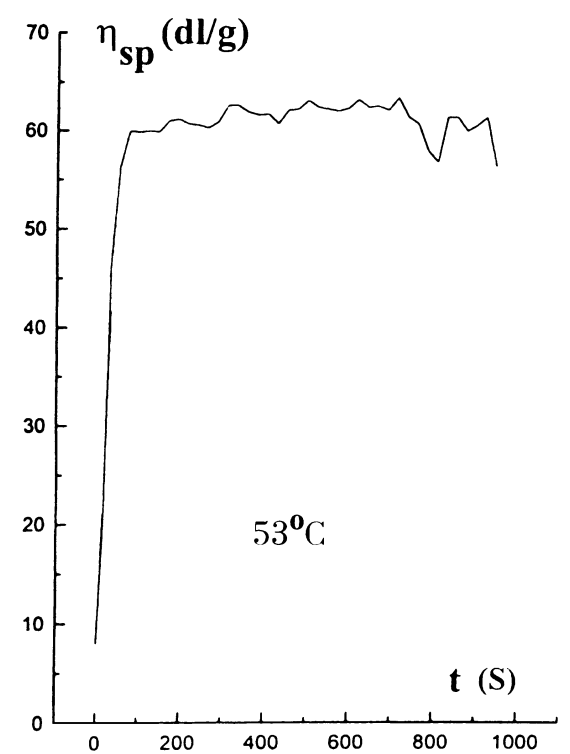

A

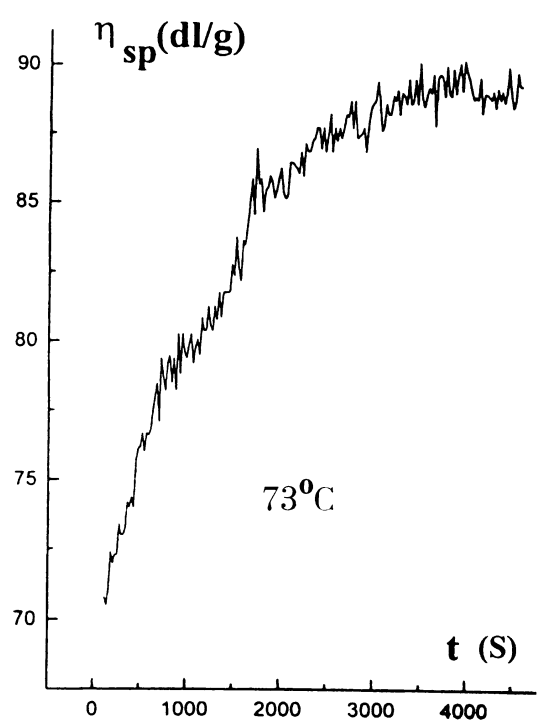

B

Fig. 5 Specific viscosity for $S_{d}$ phage suspensions vs. time, at 60 and $70{ }^{\circ} \mathrm{C}$.
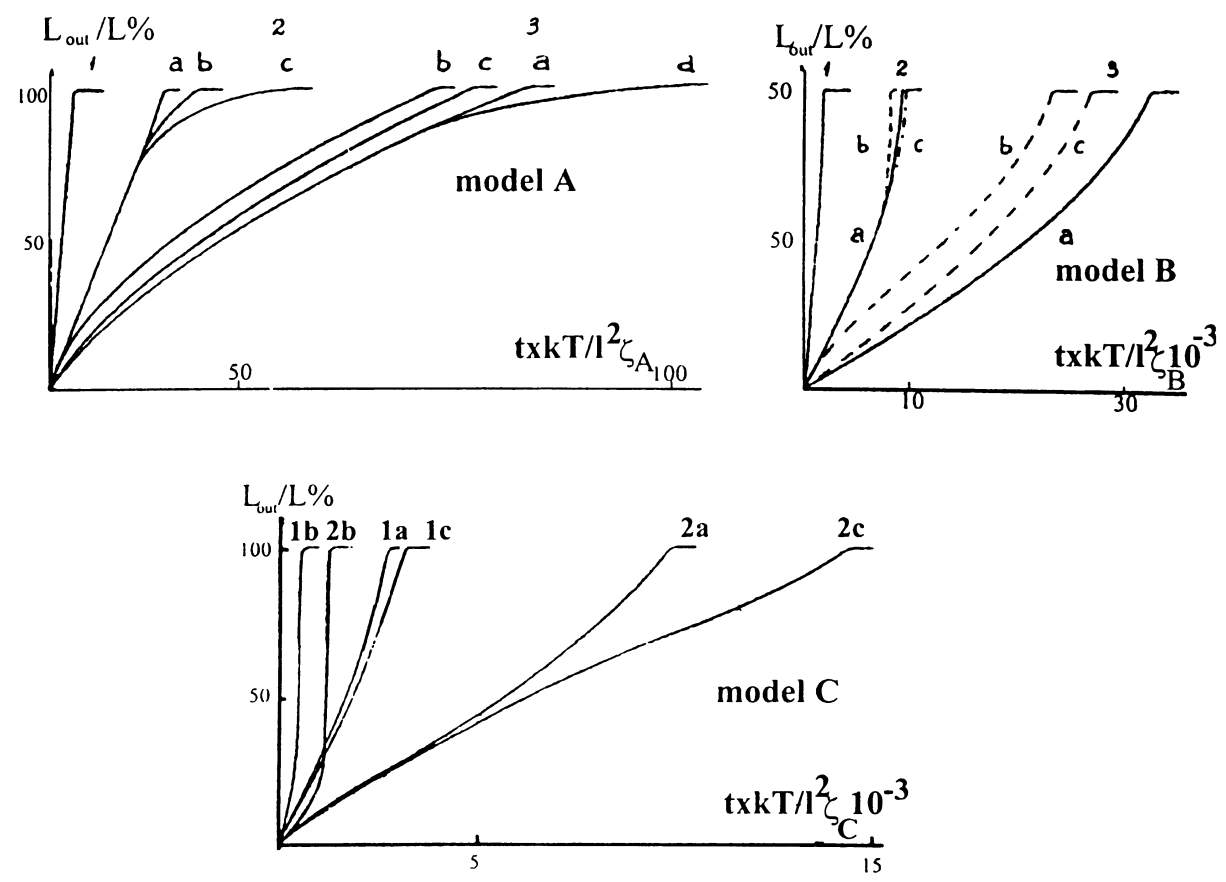

Fig. 6 Mathematical models of the time dependence of the released of ds-DNA $\left(\mathrm{L}_{\mathrm{out}} / \mathrm{L}\right)(\%)$ from phage head (according [19,20]; see also [27] ). $\mathrm{L}_{\text {out }}$ released DNA length, $\mathrm{L}$ total contour length of DNA. Model A: $1 . \Delta \tau=100, \sigma=0 ; 2 . \Delta \tau=20$, (a) $\sigma=0$, (b) $\sigma=+10$, (c) $\sigma=+5 ; 3 . \Delta \tau=5$ (a) $\sigma=0$; (b) $\sigma=-25$ (c) $\sigma=-5$ (d) $\sigma=+5$. Model B: $1 . \Delta \tau=100, \sigma=0 ; 2 . \Delta \tau=20$, (a) $\sigma=0$, (b) $\sigma=-25$, (c) $\sigma=+15 ; 3 . \Delta \tau=5$, (a) $\sigma=0$, (b) $\sigma=-25$, (c) $\sigma=-5$. Model C: $1 . \Delta \tau=20$, (a) $\sigma=0$, (b) $\sigma=-5$, (c) $\sigma=+5 ; 2 . \Delta \tau=5$ (a) $\sigma=0$, (b) $\sigma=-5$, (c) $\sigma=+5$. 


\section{Dynamic and kinetic of reptation of the ds-DNA (comparison of the experimental and the theoretical data)}

As suggested in $[19,20]$, and we see experimentally, the DNA ejection from the phage is a gradual process and is not realized by the principle of all-or-none transition: the phage nucleic acid can move ('reptate') through the channel (bacteriophage's tail tube with diameter in the range of 2-5 nm) only as a one duplex string crowling along its own axis. So the process of reptation can describe by using the equation $[19,20]$ :

$\mathrm{dF} / \mathrm{dL}_{\text {in }}=\zeta \times \mathrm{dL}_{\text {in }} / \mathrm{dt}$,

where $F$ is the free energy of system, $L_{\text {in }}$ the DNA length inside the phage at the moment $t,\left(-d F / d L_{\text {in }}\right)$ the 'moving force' of reptation-practically give information about lose the free energy (F) of the DNA; $\left(\mathrm{dL}_{\mathrm{in}} / \mathrm{dt}\right)$ the velocity of the process, $\xi$ the friction coefficient.

The most important is the question about the nature of the friction coefficient $(\xi)$. Here possible three special circumstances: (A) maximum friction is occurred in the channel of tail: $\xi$-Const; (B) in friction participate all of interphage DNA, $\xi \sim \mathrm{L}_{\mathrm{in}}$; (C) In the process of the decondensation of nucleic acid only one surface layer of the 'spool' participates in friction with internal surface of the capsid: $\xi \sim \mathrm{L}_{\text {sur }}$.

Certainly, in real systems functioning simultaneously all of this three mechanisms of friction $[19,20,27]:$

$\xi=\xi_{\mathrm{A}}+\beta \xi_{\mathrm{B}} \mathrm{L}_{\text {in }}+\gamma \xi_{\mathrm{C}} \mathrm{L}_{\text {out }}$,

where coefficients $\beta$ and $\gamma$ determined by using the character of the process of transformation of DNA out of the globular state. Different hypothesis is discussed in literature: (a) rotation of the globule of DNA as whole (so call model of 'rotation whole spool'); for this model $\gamma=1$ and $\beta$ close to zero, (b) the collective moving DNA molecule in the whole volume of globule ('moving model of ejection'; for this model $\beta=1$ and $\gamma$ close to zero). But friction in tail of phage present in all cases and is proportional to the tail length $[19,20]$.

Decrease in DNA free energy guarantees the relaxation of DNA from interphage globule to coil outside the phage particle, according to the theory and can be determined by difference in the 'solvent quality' $\Delta \tau=\tau_{\text {eff }}-\tau$ (when $\tau_{\text {eff }}$ and $\tau$ is the solvent quality in the moment of 'opening' of the channel in the tail and in the moment of packing of DNA inside the head, respectively. Theory predicts three qualitatively different characters of the dynamics of DNA reptation for three above mentioned cases [19].

Figure 6 represents the time dependence of the process of output of DNA molecule calculated according to the models A, B and C. We see that only model A is in a good agreement with the experimental data. Thus, kinetic of change of the viscosity at first step $\left(60^{\circ} \mathrm{C}\right)$, demonstrates more fast reptation of DNA and fast increase the viscosity (Fig. 5). This corresponds the high value of $\Delta \tau=100$ and demonstrates the absence of interaction of DNA with interior surface of the phage capside $(\sigma=0)$ (curve 1 Fig. 6). On the contrary kinetic of the viscosity at the second step of ejection $\left(70^{\circ} \mathrm{C}\right)$ is in good agreement with the dependence $3 \mathrm{~d}$ (Fig. 6). This coresponds with the case when $\Delta \tau$ decreases 20 -fold (!) $(\Delta \tau=5)$ and the interaction with the capside surface $(\sigma=+5)$. This demonstrates the strong interaction of DNA with the proteins of phages. The fact that this interactions definitely exists, we demonstrate by studying the completely reversible denaturation process of DNA in the presence of the phage particles (see. Fig. 2b).

Finally, it is important to obtain information about the kinetic behavior of the specific viscosity for the phages with longest tail (for example the phages DDV1 or T2-T4).

\section{CONCLUSIONS}

1 ds-DNA output from capsid, induced by temperature, is not accompanied by any thermal (endo- or exo-) heat effects (temperature interval $48-60^{\circ} \mathrm{C}$; DSC sensitivity $0.4 \mu \mathrm{W} / \mathrm{cm}$ ). So the temperature induced 'injection' of the genetic material of phages has an entropic nature or in this process totally compensates the thermal effects with opposite sign. In both cases the Nature selects the way with minimal energetic cost for injection of the genetic material of the viruses into the cell.

2 ds-DNA output from capsid, increases the viscosity of the suspension at least 100-fold (!) and recent 
rheology experiments reveal that it is not one-step process; we have at least two distinct phases of outlet of the genetic material, manifesting, that there are a strong interactions between the DNA sites and some proteins inside the capsid. It is not excluded that kinetic factors also regulate in this ejection process.

3 The contraction of the tail of phage play role in the injection probably only in the step when phage attaches to the outer membrane of host cell-the modification of virus particle takes place: structural changes in basal plate, release from 'cork'. The working hypothesis concerning physical mechanisms of injection can be formulated: The main factors of the DNA condensation and packaging in the virus head and after its ejection are caused by the surrounded solution 'quality' and so-called 'hydration forces' between ds-DNA parallel packaged segments, and more exactly, by the difference of this parameters inside and outside the capsid of the phage.

\section{REFERENCES}

1 J. D. Watson, N. H. Hopkins, J. W. Roberts, J. A. Steitz, A. M. Weiner. Molecular Biology of the Gene, 4th edn, Vol. 1, p. 185. The Benjamin/Cummings Publishing Co. Inc. (1987).

2 G. M. Mrevlishvili, T. D. Mdzinarashvili, T. N. Suladze, I. A. Andriashvili. Biophysics (Russ) 35(N2), 263 (1990).

3 G. M. Mrevlishvili, I. A. Andriashvili, D. G. Tushishvili, T. D. Mdzinarashvili, L. T. Tsinadze, N. O. Metreveli, L. G. Kalandarishvili. Biophysics (Russ) 37(N1), 48 (1992).

4 W. C. Earnshaw, S. C. Harrison. Nature 268, 598 (1977).

5 A. T. Dembo, V. V. Tikhonichev. Kristallografia (Russ). 28, 1105 (1983).

6 K. E. Richards, R. C. Williams, R. Calendar. J. Mol. Biol. 78, 255 (1973).

7 S. M. Klimenko, T. I. Tikhonenko, V. M. Andreev. J. Mol. Biol. 23, 523 (1967).

8 Y. Fujiyoshi, H. Yamagishi, T. Kinisada, H. Sugisaki, T. Kobayashi, N. M. Ueda. J. Ultrastructure Res. 79, 235 (1982).

9 G. J. Thomas, L. Yinglin, M. T. Fuller, J. King. Biochemistry 21, 3866 (1982).

10 A. T. Dembo, E. N. Dobrov, V. V. Lednev, T. I. Tikhonenko, L. A. Feigin. Biofisika (Russ) 10, 404 (1965).

11 T. I. Tikhonenko, E. I. Budovsky, V. B. Skladneva, I. S. Khromov. J. Mol. Biol. 55, 535 (1971).

12 B. P. Ulanov, T. I. Motorina, E. M. Gonokberg. Mol. Biol (Russ). 14, 390 (1980).

13 G. A. Gries, P. Serwer, P. Horowitz. Biopolymers. 24, 1635 (1985).

14 K. L. Aubrey, S. R. Casjens, G. J. Thomas. Biochemistry. 31, 11835 (1992).

15 P. Server. J. Mol. Biol. 190, 509 (1983).

16 J. Lepault, J. Dubochet, W. Baschong, E. Kellenberger. EMBO J. 6, 1507 (1987).

17 L. W. Black, J. W. Newcomb, L. W. Boring, J. C. Brown. Proc. Natl Acad. Sci. USA 82, 7960 (1985).

18 L. W. Black, M. K. Showe, A. C. Steven. In Molecular Biology of Bacteriophage T4 (J. D. Karam, ed.), pp. 218-258. American Soc. Microbiol. Press, Washington (1994).

19 I. Gabashvili, A. Grosberg, D. Kuznetcov, G. Mrevlishvili. Biophysics (Russ) 36, 788 (1991).

20 I. Gabashvili, I. Grosberg. J. Biomolecular Structure Dynamics 5, 705 (1993).

21 P. L. Privalov, V. V. Plotnicov. Thermochim. Acta 139, 257 (1989).

22 Y. U. Lyubchenko, M. D. Frank-Kamenetskii, A. V. Vologodskii, Yu. S. Lazurkin, G. G. Gause Jr. Biopolymers. 15, 1019 (1976).

23 J. Dobrov, N. A. Orareva, A. A. Manykin, T. I. Tikhonenko. Voprosi Medicinskoi Khimii (Russ) 22(N1), 98 (1976).

24 W. Senger. Principles of Nucleic Acid Structure. Springer, NY/Tokyo (1987).

25 J. W. Schwabe. Curr. Opin. Struct. Biol. 7, 126 (1997).

26 D. C. Rau, B. Lee, V. A. Parsegian. Proc. Natl Acad. Sci. USA 81, 2621 (1984).

27 I. Gabashvili. Theoretical investigation of the process of packaging and ejection of the genome of bacterial viruses. Doctoral Thesis, Tbilisi State University (1991). 